\title{
Field-Induced Formation of Nanometer-Sized Water Bridges
}

\author{
Sacha Gómez-Moñivas and Juan José Sáenz \\ Departamento de Física de la Materia Condensada and Instituto "Nicolás Cabrera," Universidad Autónoma de Madrid, \\ E-28049 Madrid, Spain \\ Montserrat Calleja and Ricardo García \\ Instituto de Microelectrónica de Madrid, CSIC, Isaac Newton 8, E-28760 Tres Cantos, Madrid, Spain
}

(Received 20 December 2002; published 30 July 2003)

\begin{abstract}
A method to form and manipulate the properties of nanometer-size liquid bridges by an external electric field is discussed. The properties of bridges are shown to be the result of an interplay among the field-induced polarization of the water layer adsorbed on the surface, the surface energy, and the water condensation from the humid air. For a given tip-sample separation, a simple model predicts the existence of a threshold voltage $V_{\text {th }}$ to form the bridge in full agreement with experiments.
\end{abstract}

DOI: 10.1103/PhysRevLett.91.056101

Developments in nanoscience and technology have emphasized the relevance of nanometer-scale solid-state metallic contacts as key elements connecting nanometer and macroscopic worlds [1]. However, despite the interest of capillary forces in many fields, such as microelectromechanical systems, magnetic hard drivers, nanolithography, or tribology, few experiments had been performed that study capillary-condensed water bridges in the nanometer range [2-5]. Liquid bridges have also played a key role in the STM imaging of poor conducting biomolecules such as DNA molecules deposited on insulating substrates [6]. Recent advances in scanning probe microscopy applications for both imaging and nanofabrication have driven new efforts to understand nanometer-size systems involving capillaries [7-10].

The electric field plays a relevant role in many systems involving capillaries, for instance the geometry of nanometer-size bridges can be modified by an external electrical field [7], however, its effects are not yet fully understood. Previous theoretical work has been focused on different aspects of the menisci formation between a tip and a surface [11], structured pores [12], capillary forces between spherical particles and substrates [13], forces between surfaces [14], and kinetics of capillary condensation $[15,16]$ in the absence of electric fields. In this Letter we analyze the field-induced formation of nanometer-size water bridges between a tip and a flat surface. We present a theoretical model together with atomic force microscopy (AFM) experimental results. The properties of bridges are shown to be the result of an interplay among the field-induced polarization of the water layer adsorbed on the surface, the surface energy, and the water condensation from the humid air. The experiments were performed with an amplitude modulation atomic force microscope operated in noncontact mode with additional circuits to apply a voltage between tip and sample [7,17]. For a given tip-sample separation, the model predicts the existence of a threshold voltage $V_{\text {th }}$ to form the bridge in agreement with experimental data.
PACS numbers: 68.37.Ps, 07.79.Lh

The behavior of $V_{\text {th }}$ versus tip-sample distance and relative humidity is also in striking agreement with the experiments.

Let us first consider a parallel plate capacitor in air with relative humidity $H$ and under an applied bias $V$ (see inset in Fig. 1). The energy $\Delta U$ required to condense a cylindrical liquid column of radius $W_{\text {neck }}$, bridging the gap $D$ between the electrodes is the sum of surface, condensation, and electrostatic contributions. The surface energy term $\Delta U_{s}$ is given by $\left(2 \pi W_{\text {neck }} D\right) \gamma_{L V}-\left(2 \pi W_{\text {neck }}^{2}\right) \times$ $\left(\gamma_{S V}-\gamma_{L S}\right)$, where the $\gamma$ 's are the energies per unit surface of the liquid-vapor $(L V)$, solid-vapor $(S V)$, and

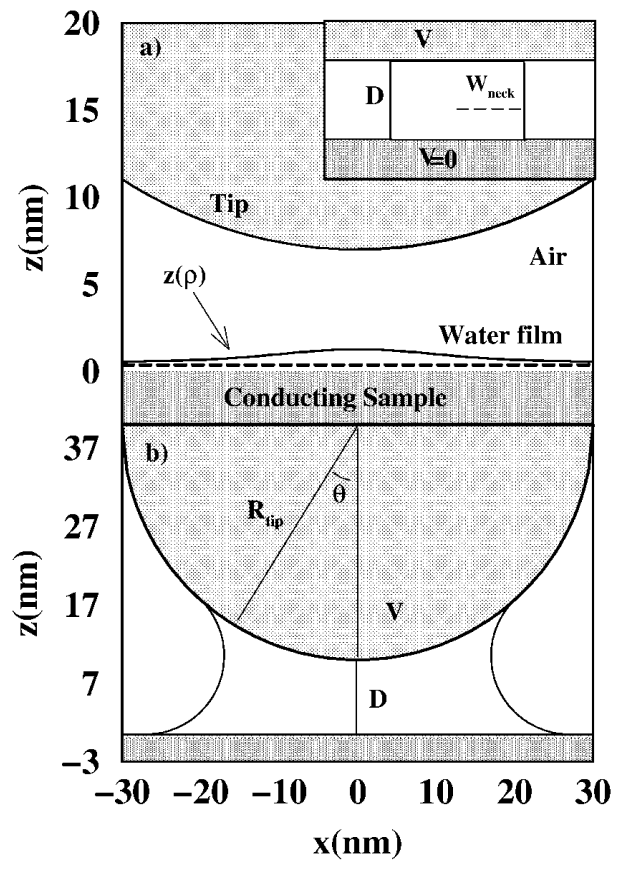

FIG. 1. (a) Tip-sample interface model. A water film wets the conducting sample. The inset represents the plane capacitor approximation. (b) Tip-sample interface after the formation of a water bridge. 
solid-liquid $(S L)$ surfaces. For simplicity, we will assume [18] $\gamma=\gamma_{L V} \approx\left(\gamma_{S V}-\gamma_{L S}\right)$ (i.e., the contact angle is taken to be $\approx 0$ ). At a given temperature $T$, the condensation energy of water molecules from air is proportional to the bridge volume $\vartheta_{o}=\pi W_{\text {neck }}^{2} D$ and is given by $\Delta U_{c} \approx\left(R T / \vartheta_{m}\right) \ln (1 / H) \vartheta_{o}$, where $R=$ $8.31 \mathrm{~J} \mathrm{~mol}^{-1} \mathrm{~K}^{-1}, \vartheta_{m}$ is the molar volume and $H$ the relative humidity. Finally, in the presence of an applied bias, the electrostatic energy $\Delta U_{e}$ associated with the polarization of the condensed liquid, is given by $-\left(\epsilon_{0} / 2\right)(\epsilon-1)(V / D)^{2} \vartheta_{o}$ with $\epsilon_{\mathrm{H}_{2} \mathrm{O}} \approx 80$. With this simple model, the energy barrier is given by

$$
\begin{aligned}
\Delta U \approx & \pi W_{\text {neck }}^{2} D\left[\frac{R T}{\vartheta_{m}} \ln (1 / H)-\frac{2 \gamma}{D}-\frac{1}{2 D^{2}}(\epsilon-1) \epsilon_{0} V^{2}\right] \\
& +2 \pi \gamma W_{\text {neck }} D .
\end{aligned}
$$

In the absence of bias, there is a critical distance $D_{C}(V=$ $0)=2 \gamma /\left[\left(R T / \vartheta_{m}\right) \ln (1 / H)\right]$ above which condensation of water is not possible (this critical distance is in agreement with the more complete model discussed in Ref. [15]). However, for any gap distance $D$ there is a threshold bias $V_{\text {th }}$ above which the most stable situation corresponds to the liquid filling the gap between the plates

$$
V_{\mathrm{th}}^{2}=\frac{1}{(\epsilon-1) \epsilon_{0}}\left\{2 D^{2} \frac{R T}{\vartheta_{m}} \ln (1 / H)-4 D \gamma\right\}
$$

Experimentally, $V_{\text {th }}$ can be determined by measuring the attractive force exerted by the water bridge on the tip. We use an amplitude modulation AFM operated in noncontact mode at resonance $(330 \mathrm{kHz})$ with additional circuits to apply a voltage between a $n^{+}$-silicon tip and a $p$-type silicon (100) sample (14 $\Omega \mathrm{cm})$ [7,17]. The silicon surface was passivated by a native oxide thin film of $1 \mathrm{~nm}$ thickness. The force on the AFM tip is detected by following the tip's oscillation as a function of time (Fig. 2): The oscillation amplitude before the application of a voltage pulse is smaller although comparable to the average tipsurface separation $D$ (region I). In Fig. 2(a) a voltage pulse of $10.4 \mathrm{~V}$ is applied for $20 \mathrm{~ms}$. The electrostatic interaction deflects the tip's equilibrium position and changes the AFM resonant frequency which leads to a reduction of the oscillation amplitude (region II). Once the pulse is off, the tip recovers its initial oscillation [Fig. 2(a)]. A different behavior is observed by applying a pulse of $11 \mathrm{~V}$ for $20 \mathrm{~ms}$. Some amplitude reduction is observed after the pulse. A liquid bridge has been formed and its capillary force reduces the oscillation. However, the meniscus is not in equilibrium and the oscillation slowly tends to its initial amplitude. At fixed $D$ and $H$, the minimum voltage that generates a pattern similar to Fig. 2(b) is identified as $V_{\text {th }}$.

The experimental results of $V_{\text {th }}$ versus tip-sample distance and relative humidity are shown in Fig. 3 (dots) together with the results of Eq. (2) (continuous line). The above model is in qualitative agreement with the experi-
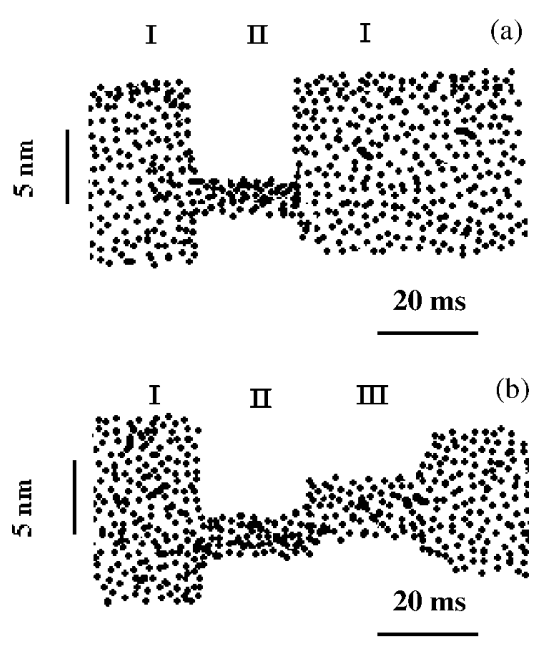

FIG. 2. Experimental tip's oscillation as a function of time for two $20 \mathrm{~ms}$ pulses, (a) $10.2 \mathrm{~V}$ and (b) $11 \mathrm{~V}$. Each point indicates the instantaneous amplitude of the tip. Because the period of the oscillation is much shorter than the pulse duration, the tip's oscillation appears as a forest of points. Successive zooms in the time axis would show the sinusoidal oscillation of the tip. During the application of the pulse (II) the tip is deflected towards the sample and the amplitude is reduced. However, in (b) some deflection and amplitude damping remains after the pulse is off (III). This is indicative of the formation of a water meniscus. $D$ and $H 8.5 \mathrm{~nm}$ and $37 \%$, respectively.

mental results: The condensation energy is increased by lowering $H$, so higher voltages are required to condense a water meniscus. On the limit of $H=100 \%$ water condenses spontaneously, and for $H=0 \%$ there are no water molecules available to form a water bridge. This has been confirmed experimentally by the application of voltages up to $24 \mathrm{~V}$. On the other hand, when the tip-sample distance is increased, electric fields under the tip decrease, and a higher voltage is necessary to induce the formation of a water bridge. However, the parallel plate capacitor model does not explain the existence of a hysteresis behavior in the dependence of the liquid bridge formation on distance and voltage. In the experiments, after turning off the voltage, a capillary force still damps the oscillation of the cantilever [Fig. 2(b)].

To fully understand the experimental data we have introduced a more realistic description of the tip-surface geometry (Fig. 1). The tip is assumed to be a sphere of radius $R_{\text {tip }}$ at a distance $D$ from a semi-infinite metallic sample. We have also considered separately two related but different processes. First, the threshold process associated with the sudden, field-induced formation of the liquid bridge. Second, the stability of the liquid bridge versus changes in the applied bias and tip-sample distance. Since the system has axial symmetry, the energy $\Delta U$ is a functional of the water surface profile $z(\rho)$ [see Fig. 1(a) $], \Delta U[z(\rho)]=2 \pi \int_{0}^{\infty} \Delta u \rho d \rho$ where $\rho$ is the radial polar coordinate on the sample surface and $\Delta u$ is the 
a)
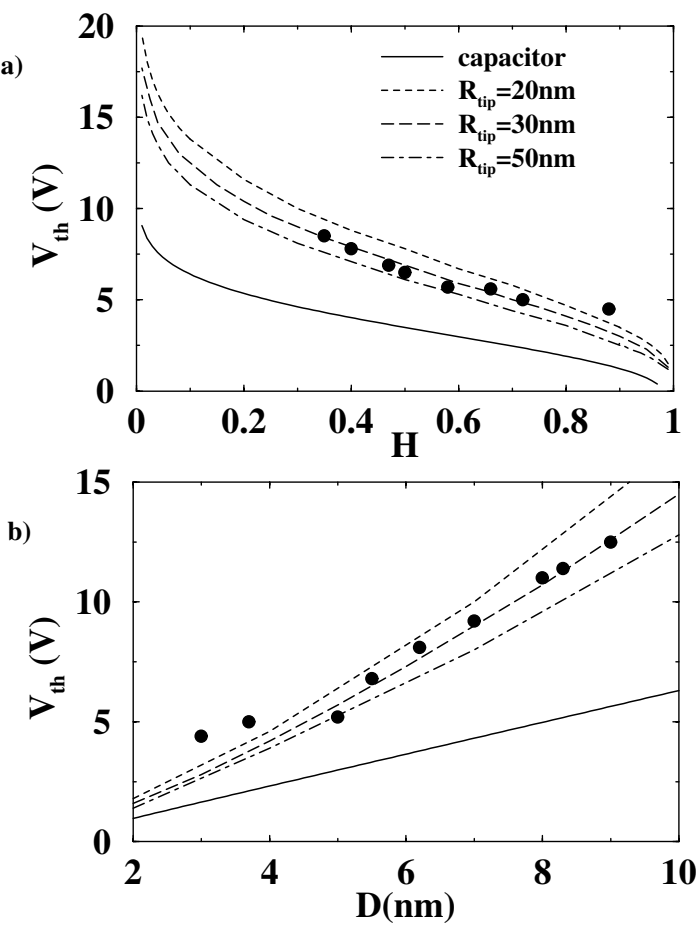

FIG. 3. Threshold voltage $V_{\text {th }}$ dependence on relative humidity $H$ (a) and tip-sample distance $D$ (b) for several radii $R_{\text {tip }}$. Continuous lines are the results of the plane capacitor approximation and solid circles are the experimental results. $D=7 \mathrm{~nm}$ in (a) and $H=30 \%$ in (b) for both theory and experiments.

energy per unit area. Taking into account the surface, condensation, and electrostatic energies together with the contribution of van der Waals forces $[18,19]$ we have

$$
\Delta u=\gamma \sqrt{1+\left(z^{\prime}\right)^{2}}+z \frac{R T}{\vartheta_{m}} \ln (1 / H)+\frac{|A|}{12 \pi z^{2}}+\Delta u_{e},
$$

where $A$ is the Hamaker constant and $z^{\prime} \equiv d z / d \rho$. Minimizing with respect to the water surface profile $(\delta \Delta U / \delta[z(\rho)]=0)$ leads to the Euler equation:

$$
\gamma \frac{\rho z^{\prime \prime}+z^{\prime}+\left(z^{\prime}\right)^{3}}{\rho\left[1+\left(z^{\prime}\right)^{2}\right]^{3 / 2}}=-\frac{|A|}{6 \pi}\left(\frac{1}{z^{3}}-\frac{1}{z_{0}^{3}}\right)+\frac{\partial \Delta u_{e}}{\partial z},
$$

where $z_{0}$ is the thickness of the water film in the absence of the tip $[18,20], z_{0}^{3}=|A| \vartheta_{m} /(6 \pi R T \ln \{1 / H\})$. This equation is valid as long as we can define a local electrostatic energy per unit area $\Delta u_{e}[z(\rho)]$. However, in general, $\Delta U_{e}$ depends on the overall shape of the surface [21]. To overcome this problem, we will consider a radial field approximation: Taking spherical coordinates (with the origin at the center of the tip) we assume that, between $\theta$ and $\theta+d \theta$ (see Fig. 1), the electric field $E(r, \theta)$ drops like in a spherical capacitor of inner radius $r_{\text {in }}=R_{\text {tip }}$ and external radius $r_{\text {ex }}=\left(D+R_{\text {tip }}\right) / \cos (\theta)$. Taking into account that $\tan (\theta) \equiv \rho /\left(D+R_{\text {tip }}\right)$, the polarization term can be written as

$$
\begin{aligned}
& \Delta U_{e}=-\frac{\epsilon_{0}}{2} \int\left[\epsilon E^{2}-E_{0}^{2}\right] d V=-2 \pi \int \Delta u_{e} \rho d \rho, \\
& \Delta u_{e}=\epsilon_{0} \frac{\sin (\theta)}{2 \rho} \frac{d \theta}{d \rho} \int_{r_{\mathrm{in}}}^{r_{\mathrm{ex}}(\theta)} r^{2} d r\left[\epsilon(r) E^{2}(r, \theta)-E_{0}^{2}\right],
\end{aligned}
$$

where $E_{0}$ is the radial electric field in the absence of water and $\epsilon(r)=\epsilon_{\mathrm{H}_{2} \mathrm{O}}$ for $r_{\mathrm{ex}}>r>\left[D+R_{\text {tip }}-z\right] / \cos (\theta)$.

The numerical solution of Eq. (4) shows that for small voltages, the electrostatic term induces a small bump of the water surface below the tip apex as shown in Fig. 1(a). The height of the bump increases with the bias. However, before its height becomes comparable with the gap distance, there is a threshold bias above which there is not stable solution without bridging the tip and sample. The dependencies of $V_{\text {th }}$ on $H, D$, and $R_{\text {tip }}$ are plotted in Fig. 3 . Excellent quantitative agreement with the experiments is obtained for $R_{\text {tip }} \approx 30 \mathrm{~nm}$. The instability approximately occurs at a given threshold field on the sample surface. This implies that for tip-sample distances $D$ smaller than the tip radius, the threshold bias increases linearly with $D$. However, for larger distances $\left(D \gg R_{\text {tip }}\right)$, most of the voltage drops in the proximity of the tip's apex and $V_{\text {th }}$ increases exponentially with $D$ [22].

In order to analyze the stability of the system once the liquid bridge has been formed, we have assumed a liquid meniscus having a constant curvature radius which smoothly joins tip and sample surfaces [Fig. 1(b)] [15]. We then evaluate the surface and condensation energies taking into account the geometry of the neck [Fig. 1(b)] while the electrostatic energy is calculated within the radial field approach. For any bias larger than a critical bias $V_{c}$, the energy versus bridge radius presents a minimum. $V_{c}$ represents a lower limit such that for $V<V_{c}$ the liquid bridge cannot exist. As expected $V_{c}$ is always smaller than $V_{\text {th }}$.

In Fig. 4 we have plotted $V_{c}$ and $V_{\text {th }}$ with respect to $\mathrm{D}$ for $R_{\text {tip }}=30 \mathrm{~nm}$ and $H=30 \%$. For a fixed bias

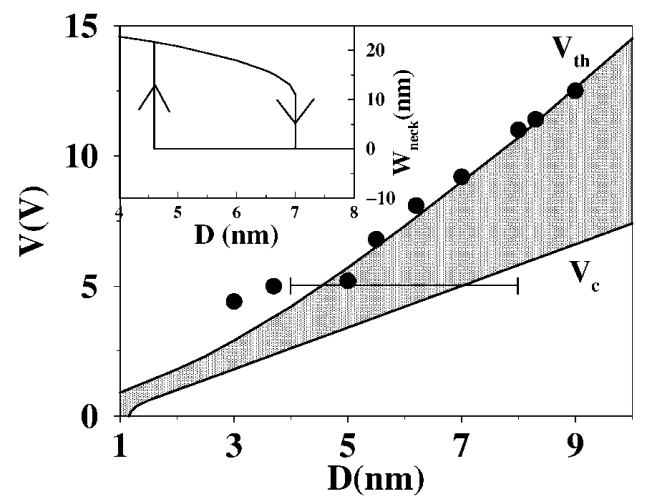

FIG. 4. $V_{\text {th }}$ and $V_{c}$ dependence on tip-sample distance $D$ ( $\left.H=30 \%, R_{\text {tip }}=30 \mathrm{~nm}\right)$. Once is has been formed, the bridge exists for voltage between $V_{\text {th }}$ and $V_{c}$ (shaded area). The inset shows a hysteresis curve, $W_{\text {neck }}$ vs $D$ at $V=5 \mathrm{~V}$ ). Solid circles represent the experimental $V_{\text {th }}$ values for $H=30 \%$. 
(tip-sample distance), there is a window of tip-sample distances (bias) in which the existence of the liquid bridge depends on the initial conditions. This is illustrated in the inset of Fig. 4. At larger separations $(V=5 \mathrm{~V})$ there is no water meniscus bridging tip and sample. As the tip approaches the sample, there is a threshold distance at which the bridge forms spontaneously. Once the bridge is formed, it remains stable up to a larger critical distance. An equivalent hysteresis occurs for a fixed distance as a function of the bias. It is worth noticing that those critical values are obtained from equilibrium arguments. However, experimentally, the slow kinetics of the condensation may have a dominant role $[15,16]$. This explains why, although the hysteresis is observed experimentally, the measured critical distances (voltages) are much larger (lower) than those predicted by the model. The divergence between theory and experiments at short distances is attributed to the presence of local asperities in the experimental tip.

In conclusion, we have presented a model of the fieldinduced formation of water bridges between a tip and a flat surface. For a given tip-sample separation, the model predicts the existence of a threshold voltage $V_{\text {th }}$ to form the bridge in agreement with the experimental results. Our results provide a method to form and manipulate the properties of nanometer-size liquid bridges by an external electric field. This could open the possibility to create tiny electrochemical reactors to perform selective chemical reactions at nanoscale.

We would like to thank J. Colchero, L. Froufe-Pérez, J. Gómez-Herrero, C. Gómez-Navarro, R. Guckenberger, and M. Tello for interesting discussions. This work has been supported by the Spanish MCyT (Refs. No. /PB980464, PB98-0471/ and No. BFM2000-1470-C02-02) and the European Commission, G5RD-CT2000-00349 (MONA-LISA).

[1] N. Agraï, A. Levy-Yeyati, and J. M. van Ruitenbeek, Phys. Rep. 377, 81 (2003); in Nanowires, edited by P. A. Serena and N. García, NATO ASI, Ser. E, Vol. 340 (Kluwer Academic Publishers, Dordrecht, Boston, 1997).

[2] L. R. Fisher and J. N. Israelachvili, J. Colloid Interface Sci. 80, 528 (1981).

[3] C. M. Mate, M. R. Lorenz, and V. J. Novotny, J. Chem. Phys. 90, 7550 (1989).

[4] M. Schenk, M. Füting, and R. Reichelt, J. Appl. Phys. 84, 4880 (1998).
[5] E. Riedo, F. Lévy, and H. Brune, Phys. Rev. Lett. 88, 185505 (2002).

[6] M. Heim, R. Eschrich, A. Hillebrand, H. F. Knapp, G. Cevc, and R. Guckenberger, J. Vac. Sci. Technol. B 14, 1498 (1996); R. Guckenberger, M. Heim, G. Cevc, H. Knapp, W. Wiegräbe, and A. Hillebrand, Science 266, 1538 (1994).

[7] R. García, M. Calleja, and F. Pérez-Murano, Appl. Phys. Lett. 72, 2295 (1998); R. García, M. Calleja, and H. Rohrer, J. Appl. Phys. 86, 1898 (1999).

[8] R. D. Piner, J. Zhu, F. Xu, S. Hong, and C. A. Mirkin, Science 283, 661 (1999).

[9] T. Thundat, X. Y. Zheng, G. Y. Chen, and R. J. Warmack, Surf. Sci. 294, L939 (1993); M. Luna, J. Colchero, and A. M. Baró, Appl. Phys. Lett. 72, 3461 (1998).

[10] K. Morimoto, K. Araki, K. Yamashita, K. Morita, and M. Niwa, Appl. Surf. Sci. 117/118, 652 (1997).

[11] C. Gao, Appl. Phys. Lett. 71, 1801 (1997); J.-Y. Yuan, Z. Shao, and C.Gao, Phys. Rev. Lett. 67, 863 (1991).

[12] A. Valencia, M. Brinkmann, and R. Lipowsky, Langmuir 17, 3390 (2001).

[13] A. de Lazzer, M. Dreyer, and H. J. Rath, Langmuir 15, 4551 (1999).

[14] R. G. Horn, J. Am. Ceram. Soc. 73, 1117 (1990).

[15] F. Restagno, L. Bocquet, and T. Bilben, Phys. Rev. Lett. 84, 2433 (2000).

[16] M. M. Kohonen, N. Maeda, and H. K. Christenson, Phys. Rev. Lett. 82, 4667 (1999).

[17] Further description of the dynamic parameters needed to operate the AFM in a noncontact mode and to measure tip-sample separation can be found in R. García and R. Pérez, Surf. Sci. Rep. 47, 197 (2002).

[18] J. Israelachvili, Intermolecular and Surface Forces (Academic Press, London, 1992).

[19] For small tip radius the short range van der Waals contribution from the tip apex is neglected in comparison with that of the flat surface. In the absence of an electrical field, this is the same approach used to analyze the stability of liquid bridges in STM [11].

[20] For some surfaces the absorption of water cannot be described by the simplest van der Waals interaction [R. M. Pashley and J. A. Kitchener, J. Colloid Interface Sci. 71, 491 (1979)]. However, the main effect of the $1 / z^{3}$ van der Waals term in Eqs. (3) and (4) is to stabilize a water film of thickness $z_{0}$ on the sample surface and it does not play a major role in determining the equilibrium shape of the neck (see also Ref. [15]).

[21] S. Gómez-Moñivas, J. J. Sáenz, R. Carminati, and J. J. Greffet, Appl. Phys. Lett. 76, 2955 (2000).

[22] G. Mesa, E. Dobado-Fuentes, and J. J. Sáenz, J. Appl. Phys. 79, 39 (1996). 